\title{
Correction to: Order-theoretic properties and separability of some sets of quasi-measures
}

\section{Zbigniew Lipecki ${ }^{1}$}

Published online: 7 September 2019

(c) The Author(s) 2019

\section{Correction to: Ricerche mat. (2018) 67:581-595 https://doi.org/10.1007/s11587-018-0373-0}

Here is a corrected version of the Abstract.

\begin{abstract}
Let $\mathfrak{M}$ and $\mathfrak{R}$ be algebras of subsets of a set $\Omega$ with $\mathfrak{M} \subset \mathfrak{R}$. Denote by $E(\mu)$ the set of all quasi-measure extensions of a given quasi-measure $\mu$ on $\mathfrak{M}$ to $\mathfrak{R}$. We present some results on the coincidence of the bands, in $b a(\Re)$, generated by $E(\mu)$ and extr $E(\mu)$. Moreover, we show that if $\mu$ is atomic, then $E(\mu)$ is contained in a principal band in $b a(\Re)$ if and only if it is separable. Another sufficient condition for the separability of $E(\mu)$ is also presented.
\end{abstract}

The corrections consist in replacing " $\Re$ " by " $b a(\mathfrak{R})$ " in lines 3 and 5 thereof.

Here are other corrections needed in the text.

1. In the fifth paragraph on p. 585, the final formula should read as follows: $\mu=$ $\sum_{v \in \mathcal{U}_{\mu}} v$.

2. Condition (i) of Proposition 4 on p. 585 should read as follows: $\operatorname{ba}(\mathfrak{M})=B_{\text {ult }}(\mathfrak{M})$.

3. In Remark 2 on p. 586, the displayed formula should read as follows:

$$
B_{p a(\mathfrak{M})}=B_{\text {ult }(\mathfrak{M})}
$$

4. The first inequality in the proof of Proposition 5 on p. 586 should read as follows: $\tau \geqslant \sum_{\pi \in \mathcal{F}} \tau_{\pi}$.

5. In Remark 3 on p. 586, " $2^{c}$ " should read as follows: " $2^{\mathfrak{c} " ~}$ twice.

The original article can be found online at https://doi.org/10.1007/s11587-018-0373-0.

Zbigniew Lipecki

lipecki@impan.pan.wroc.pl

1 Institute of Mathematics, Polish Academy of Sciences, Wrocław Branch, Kopernika 18, 51-617 Wrocław, Poland 
6. In the line following the proof of Theorem 3 on p. 592, "equivalence of" should read "equivalent".

7. In the first paragraph on p. 593, "a cardinality $\geq 1$ " should read "a cardinal $\geq 1$ ".

Open Access This article is distributed under the terms of the Creative Commons Attribution 4.0 International License (http://creativecommons.org/licenses/by/4.0/), which permits unrestricted use, distribution, and reproduction in any medium, provided you give appropriate credit to the original author(s) and the source, provide a link to the Creative Commons license, and indicate if changes were made.

Publisher's Note Springer Nature remains neutral with regard to jurisdictional claims in published maps and institutional affiliations. 